\title{
Article
}

\section{Phoebe 2.0 - Triple and multiple systems}

Pavlovski, K., Conroy, K., Degroote, P., Hambleton, Kelly, Bloemen, S., Pablo, H., Giammarco, J., Prša, A., Tkachenko, A. and Torres, G.

Available at http://clok.uclan.ac.uk/14566/

Pavlovski, K., Conroy, K., Degroote, P., Hambleton, Kelly, Bloemen, S., Pablo, H., Giammarco, J., Prša, A., Tkachenko, A. et al (2013) Phoebe 2.0 - Triple and multiple systems. EAS Publications Series, 64 . pp. 295-298. ISSN 1633-4760

It is advisable to refer to the publisher's version if you intend to cite from the work. http://dx.doi.org/10.1051/eas/1364040

For more information about UCLan's research in this area go to http://www.uclan.ac.uk/researchgroups/ and search for < name of research Group>.

For information about Research generally at UCLan please go to http://www.uclan.ac.uk/research/

All outputs in CLoK are protected by Intellectual Property Rights law, including Copyright law. Copyright, IPR and Moral Rights for the works on this site are retained by the individual authors and/or other copyright owners. Terms and conditions for use of this material are defined in the policies page.

\section{CLoK}

Central Lancashire online Knowledge www.clok.uclan.ac.uk

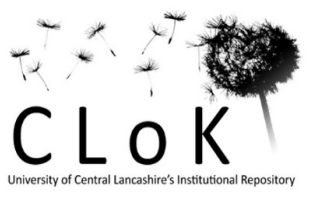




\title{
PHOEBE 2.0 - TRIPLE AND MULTIPLE SYSTEMS
}

\author{
K. Conroy ${ }^{1}$, P. Degroote ${ }^{2}$, K. Hambleton ${ }^{3}$, S. Bloemen ${ }^{4}$, H. Pablo ${ }^{5}$, \\ J. Giammarco ${ }^{6}$ and A. Prša ${ }^{7}$
}

\begin{abstract}
Some close binary formation theories require the presence of a third body so that the binary orbit can shrink over time. Tidal friction and Kozai cycles transfer energy from the binary to its companion, resulting in a close inner binary and a wide third body orbit. Spectroscopy and imaging studies have found $40 \%$ of binaries with periods less than 10 days, and $96 \%$ with periods less than 3 days, have a wide tertiary companion. With recent advancements in large photometric surveys, we are now beginning to detect many of these triple systems by observing tertiary eclipses or through the effect they have on the eclipse timing variations (ETVs) of the inner-binary. In the sample of 2600 Kepler EBs, we have detected the possible presence of a third body in $\sim 20 \%$, including several circumbinary planets. Some multiple systems are quite dynamical and feature disappearing and reappearing eclipses, apsidal motion, and large disruptions to the inner-binary. PHOEBE is a freely available binary modeling code which can dynamically model all of these systems, allowing us to better test formation theories and probe the physics of eclipsing binaries.
\end{abstract}

\section{Introduction}

Using transit timings and eclipse timings to find exoplanets is a well-known method (Schwarz et al. 2011). With Kepler data alone, Fabrycky (2012), Ford (2012), and

\footnotetext{
${ }^{1}$ Department of Physics and Astronomy, Vanderbilt University, VU Station B 1807, Nashville, TN 37235, USA

2 Instituut voor Sterrenkunde, KU Leuven, Celestijnenlaan 200D, 3001 Leuven, Belgium

3 Jeremiah Horrocks Institute, University of Central Lancashire, Preston PR1 2HE, UK

${ }^{4}$ Department of Astrophysics, Radboud University Nijmegen, Heyendaalseweg 135, 6525 AJ Nijmegen, The Netherlands

5 Département de Physique, Université de Montréal, CP 6128, Succursale Centre-Ville, Montréal, QC, H3C 3J7, Canada

${ }^{6}$ Department of Astronomy and Physics, Eastern University, Saint Davids, PA 19087, USA

${ }^{7}$ Department of Astrophysics and Planetary Sciences, Villanova University, 800 E Lancaster Ave, Villanova, PA 19085, USA
} 
Steffen (2012) have used transit timings to detect and study multiple planetary systems. Kepler 16 (Doyle et al. 2011), 34, and 35 (Welsh et al. 2012) were validated, in part, through their eclipse timing variations.

Furthermore, approximately $20 \%$ of Kepler eclipsing binaries have third-body candidates (Conroy et al. 2013; Rappaport et al. 2013; Gies et al. 2012; Orosz et al., in preparation), most of which are likely stellar companions. Only a few of these have been confirmed with tertiary transits, as the third-body is often on a very wide orbit and must be perfectly aligned in order to observe eclipse events.

Modeling triple systems can often allow for greater precision in the resulting fundamental parameters than eclipsing binaries without companions. By modeling a large sample of multiple systems we can test theories of close binary formation and planetary migration caused by the presence of a third body, such as Kozai cycles and tidal friction (Bonnell 2001; Kiseleva et al. 1998).

\section{Modeling ETVs}

Borkovits et al. (2011) determined analytic functions for the "light time travel effect" (LTTE) component of the ETV signal. If the orbit of the third body is wide enough compared to the inner-binary, then dynamical effects can be ignored, leaving us with the following expression for the timings:

$$
E T V_{\mathrm{LTTE}}=A_{\mathrm{LTTE}}\left[\left(1-e_{3}^{2}\right)^{1 / 2} \sin E_{3}(t) \cos \omega_{3}+\left(\cos E_{3}(t)-e_{3}\right) \sin \omega_{3}\right]
$$

where

$$
\begin{gathered}
E_{3}(t)=M_{3}(t)+e_{3} \sin E_{3}(t) \\
M_{3}(t)=\left(t-t_{0}\right) \frac{2 \pi}{P_{3}} \\
A_{\mathrm{LTTE}}=\frac{G^{1 / 3}}{c(2 \pi)^{2 / 3}}\left[\frac{m_{3}}{m_{123}^{2 / 3}} \sin i_{3}\right] P_{3}^{2 / 3}
\end{gathered}
$$

where $t_{0}$ is a time offset, $m_{3}$ is the mass of the third body, $m_{123}$ is the mass of the entire system, and $P_{3}, i_{3}, e_{3}, \omega_{3}, E_{3}(t)$, and $M_{3}(t)$ are the period, inclination, eccentricity, argument of periastron, eccentric anomaly, and mean anomaly of the third body orbit, respectively.

Without any information on the inclination of the third body, we can fit $A, e_{3}$, $P_{3}$, and $\omega_{3}$. Along with $P_{3}, A$ includes $\frac{m_{3}}{m_{123}^{23}} \sin i_{3}$, but we cannot get the mass of the third body without both the inclination and mass of the inner-binary.

\subsection{Modeling ETVs with tertiary eclipses}

In cases where we observe the eclipse of the third body, we can constrain $i_{3}$ to be near $90^{\circ}$. This gives us $\frac{m_{3}}{m_{123}^{2 / 3}}$ as well as the mutual inclination between the two orbits. If the inner-binary is an ellipsoidal variable (perhaps not eclipsing but 

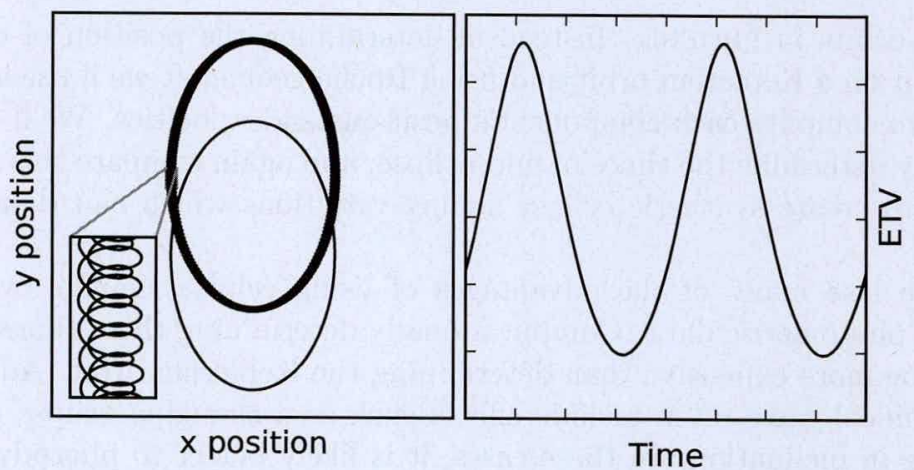

Fig. 1. Top-down view of a hierarchical orbit (left) with inset showing the orbit of the inner-binary. The companion causes the barycenter of the inner-binary to move, resulting in a light time delay in the observations of the eclipse times. The synthetic eclipse timing variations (right) can then be used to fit the orbit of a third body to data.

causing a sinusoidal like signal due to tidal distortion), then we can measure these large mutual inclinations and use these systems to test the predictions of Kozai cycles and tidal friction as a method of close binary formation.

\section{Implementation in PHOEBE 2.0}

\subsection{Hierarchical}

PHOEBE 2.0 allows for creating a hierarchical system, such that a component of a binary can be another binary itself. The orbital dynamics and lightcurve creation are treated the same as a traditional binary. If light time effects are enabled, eclipse times will be modeling including this delay effect.

Eclipse timing variations themselves can also be modeled and fit. We compute the Keplerian orbits of all components and determine the barycentric times at which each eclipse will occur. By comparing this to the linear ephemeris, we can simulate eclipse timing variations (Fig. 1).

Fitting to eclipse timing variations over the original photometric data can have several advantages. First, since this is all done analytically and does not require integrating over the mesh, this operation is much cheaper than synthetically creating the entire lightcurve and fitting each eclipse to the eclipse data. Secondly, especially in older datasets, often only eclipse times are reported. In these cases, modeling the eclipse times allows the model to extend to a much longer time baseline than would be the case if only using recent photometric data.

\subsection{Dynamical}

To deal with dynamical multiple systems in which the inner binary is actually perturbed by an outer body, we plan to implement an N-body alternative for 
computing orbits in PHOEBE. Instead of determining the position of each mesh by placing it on a Keplerian orbit and using Roche geometry, we'll use an N-body integrator to compute each component's positions and velocities. We'll then computationally determine the times of mid-eclipse, and again compare this to a linear ephemeris to create synthetic eclipse timing variations which can then be fit to data.

Here we lose many of the advantages of using eclipse timings over relying entirely on photometric data. Computationally determining these times of eclipse will likely be more expensive than determining the Keplerian orbit. Additionally, these dynamical cases often include effects such as a changing eclipse depth due to a change in inclination. In these cases, it is likely better to photodynamically model the entire lightcurve if possible.

\section{Discussion}

PHOEBE 2.0 introduces several new datatypes for modeling and fitting, including eclipse timing variations. We can now model and fit photometric, spectroscopic, radial velocity, and eclipse timing data simultaneously. Doing so will allow modeling these triple and multiple systems by utilizing information from tertiary eclipses, triple lined spectra, and variations in the eclipse timings due to the presence of additional bodies.

Modeling these systems in detail will help us to further constrain stellar parameters and test predictions of close binary formation theories.

\section{References}

Bonnell, I.A., 2001, in The Formation of Binary Stars, Proc. IAU Symp., 200, 23

Borkovits, T., Csizmadia, S., Forgács-Dajka, E., \& Hegedüs, T., 2011, A\&A, 528, A53

Conroy, K.E., Prša, A., Stassun, K.G., et al., 2013, AJ, in press [arXiv: 1306.0512]

Doyle, L.R., Carter, J.A., Fabrycky, D.C., et al., 2011, Science, 333, 1602

Fabrycky, D.C., Ford, E.B., Steffen, J.H., et al., 2012, ApJ, 750, 114

Ford, E.B., Ragozzine, D., Rowe, J.F., et al., 2012, ApJ, 756, 185

Gies, D.R., Williams, S.J., Matson, R.A., et al., 2012, AJ, 143, 137

Kiseleva, L.G., Eggleton, P.P., \& Mikkola, S., 1998, MNRAS, 300, 292

Rappaport, S., Deck, K., Levine, A., et al., 2013, ApJ, 768, 33

Schwarz, R., Haghighipour, N., Eggl, S., et al., 2011, MNRAS, 414, 2763

Steffen, J.H., Ford, E.B., Rowe, J.F., et al., 2012, ApJ, 756, 186

Welsh, W.F., Orosz, J.A., Carter, J.A., et al., 2012, Nature, 481, 475 
Copyright of EAS Publications Series is the property of Cambridge University Press and its content may not be copied or emailed to multiple sites or posted to a listserv without the copyright holder's express written permission. However, users may print, download, or email articles for individual use. 